\title{
REMARKS ON THE INTRINSIC INVERSE PROBLEM
}

\author{
DANIEL BERTRAND \\ Institut de Mathématiques de Jussieu, Case 247 \\ F-75252 Paris Cédex 05, France \\ E-mail: bertrand@math.jussieu.fr
}

\begin{abstract}
The intrinsic differential Galois group is a twisted form of the standard differential Galois group, defined over the base differential field. We exhibit several constraints for the inverse problem of differential Galois theory to have a solution in this intrinsic setting, and show by explicit computations that they are sufficient in a (very) special situation.
\end{abstract}

1. The intrinsic differential Galois group. Let $(K, \partial)$ be a differential field of characteristic 0 with algebraically closed constant field $C$, let $\mathcal{D}_{\mathcal{K}}$ be the ring of differential operators $K[\partial]$ and let $\mathbf{D}_{K}$ be the tannakian category formed by the left $\mathcal{D}_{\mathcal{K}}$-modules $M$, whose underlying $K$-vector space $V$ has finite dimension. Fix an object $M$ in $\mathbf{D}_{K}$ (reference to $M$ will often be omitted in the notations).

Let $\omega$ be a fiber functor of $\mathbf{D}_{K}$ over $C$ (cf. [Br], 1.1.5), and let $G^{\omega}(M)$, or $G^{\omega}$ for short, be the quotient of the $C$-proalgebraic group $A u t^{\otimes}(\omega)$ cut out by $M$; the $C$-points of $G^{\omega}$ form the usual differential Galois group of the Picard-Vessiot extension $L=L_{M, \omega}$ of $K$ defined by $M$ at $\omega$. By extension of scalars from $C$ to $K$, we obtain a $K$-group $G_{K}^{\omega}:=G^{\omega}(M) \otimes_{C} K$. (In [B], these groups were respectively denoted by $G_{C}$ and $G_{K}$.)

If we replace $\omega$ by the the forgetful fiber functor $M \rightarrow V$ of $\mathbf{D}_{K}$, we obtain instead of $G^{\omega}$ an algebraic group $G^{K}(M)=G^{K}$ over $K$ : the stabilizer in $G L(V)$ of all the objects of $\mathbf{D}_{K}$ which occur as subquotients in sums of tensor powers involving $M$ and its dual. This group, first considered in [K1] for arithmetic purposes, can be called the intrinsic differential Galois group of $M$ (as in [A1]), or the Katz group (as in $[\mathrm{P}]$ ). When $K=C(B)$ is the function field of an algebraic variety $B$ (cf. [K1], [A2]), it may also be viewed as the Galois group based at the generic point of $B$, and called the generic differential Galois group of $M$. (In $[\mathrm{B}]$ and $[\mathrm{P}]$, it is denoted by $G_{K}^{\prime} \cdot$ )

We now recall the existence of an algebraic variety $\mathcal{P}^{K, \omega}=\mathcal{P}^{K, \omega}(M)$ over $K$, whose field of $K$-rational functions is isomorphic to the Picard-Vessiot extension $L_{M, \omega}$, and which is, in a compatible way, both a left torsor under $G^{K}$ and a right torsor under

2000 Mathematics Subject Classification: Primary 12H05; Secondary 20 G15.

The paper is in final form and no version of it will be published elsewhere. 
$G_{K}^{\omega}$. In particular, $G^{K}$ and $G_{K}^{\omega}$ become isomorphic over the algebraic closure $\bar{K}$ of $K$ : more precisely, the bitorsor structure on $\mathcal{P}^{K, \omega}$ shows that $G^{K}$ is an inner $K$-form of $G_{K}^{\omega}$ (cf. [K1], 4.1, [Br] , 5.2). Since the connected component of the identity $\left(G^{K}\right)^{0}$ of $G^{K}$ is defined over $K$, it too is a $K$-form of the connected component of the identity $\left(G^{\omega}\right)^{0} \otimes_{C} K=\left(G_{K}^{\omega}\right)^{0}$ of $G_{K}^{\omega}$, though usually not an inner one anymore (see $\S 3$ below for several examples).

Let $\Gamma_{K}=\operatorname{Gal}(\bar{K} / K)$ be the absolute Galois group of our base field $K$, and fix a $K$-group $G$ with uninteresting $K$-structure (for instance, $K$-split, as is $G_{K}^{\omega}$ ). The isomorphism classes of the $K$-torsors over $G$ (resp. the $K$-forms of $G$ ) are parametrized by the pointed set $H^{1}\left(\Gamma_{K}, G(\bar{K})\right)$ (resp. $H^{1}\left(\Gamma_{K}, A u t_{\bar{K}}(G)\right)$ ), where $A u t_{\bar{K}}(G)$ denotes the group of $\bar{K}$-automorphisms of $G$. The classes of inner forms of $G$ lie in the image of $H^{1}\left(\Gamma_{K}, G(\bar{K})\right)$ under its natural (usually not injective) map Int to $H^{1}\left(\Gamma_{K}, A u t_{\bar{K}}(G)\right)$, cf [Sp], 12.3.7. The knowledge of $G^{K}(M)$ is therefore a priori finer than that of $G^{\omega}(M)$, but less precise than that of $\mathcal{P}^{K, \omega}$. One of the problems we raise here is where in between it really lies: cf. end of $\S 3$. Another problem we address is whether each 'reasonable' $K$-form of a given $K$-group $G$ takes the shape of a $G^{K}(M)$ for some $M$ : cf. $\S 2$, and some explicit examples in $\S 3$. In neither cases do we get definitive answers, and the paper should just be viewed as a random approach to a better formulation of these problems.

Of course, such questions are of interest only if $K$ is not algebraically closed, and for a given $M \in \mathbf{D}_{K}$, the algebraic closure $K^{\prime}:=K_{M, \omega}^{\prime}$ of $K$ in the Picard-Vessiot extension $L_{M, \omega}$ plays a role in our study. Denoting by $\omega^{\prime}$ a fiber functor over $C$ extending $\omega$ to $\mathbf{D}_{K^{\prime}}$, we recall that $L / K^{\prime}$ is a regular Picard-Vessiot extension, whose standard differential Galois group is the group of $C$-points of the connected $C$-group $G^{\omega^{\prime}}\left(M \otimes_{K}\right.$ $\left.K^{\prime}\right) \simeq\left(G^{\omega}(M)\right)^{0}$, while on the level of intrinsic groups (now over $K^{\prime}$ ), we have in view of [K 1], Prop. 4.3,

$$
G^{K^{\prime}}\left(M \otimes_{K} K^{\prime}\right)=\left(G^{K}(M)\right)^{0} \otimes_{K} K^{\prime} .
$$

Thus, the (classical) Galois extension $K^{\prime} / K$ has intrinsic Galois group $X^{K}:=G^{K} /\left(G^{K}\right)^{0}$; this amounts to endowing the finite group $X^{\omega}:=G^{\omega} /\left(G^{\omega}\right)^{0} \simeq \Gamma_{K} / \Gamma_{K^{\prime}} \simeq \operatorname{Gal}\left(K^{\prime} / K\right)$ with the structure of $\Gamma_{K}$-group given by conjugation by the elements of $\Gamma_{K}$.

Finally, two points on terminology:

- $K$-split ${ }^{(1)}$ group $G$ : although the intrinsic Galois groups $G^{K}(M)$ come equipped with a connexion, this expression is here not used in the sense of Pillay's paper $[\mathrm{P}]$ in these Proceedings, but in the usual sense of algebraic groups over fields of characteristic 0 , namely: a $K$-group is $K$-split if its connected component of the identity contains a maximal torus $T$ which is defined over $K$ (there always is one) and whose group of characters over $K$ generates the $K$-algebra $K[T]$. Any $K$-group $G$ is split over $\bar{K}$, and a minimal subfield of $\bar{K}$ over which $G$ is split will be called a minimal splitting field for $G$.

- constant $K$-group $G$ : this just means that $G$ is obtained from a $C$-group by extension of scalars from $C$ to $K$ (typical example: $G=G_{K}^{\omega}$ ). Since $C$ is algebraically closed, a

${ }^{(1)}$ i.e. groupe déployé sur $K$. We shall also encounter split extensions (extensions scindées), but fortunately no splitting field of a polynomial (corps de décomposition) or of an algebra (corps neutralisant). 
constant $K$-group is trivially split over $K$. A constant finite group $X$ (typical example: $\left.X^{\omega}:=G^{\omega} /\left(G^{\omega}\right)^{0}\right)$ is the finite $\Gamma_{K}$-group corresponding to the trivial action of $\Gamma_{K}$ on $X$.

2. The intrinsic inverse problem. Let $(K, \partial)$ be a differential field as in $\S 1$, let $G$ be a $K$-algebraic group, and let $R: G \rightarrow G L(V)$ be a faithful $K$-rational representation on a $K$-vector space $V$ of finite dimension. In [B], I raised the following 'intrinsic' version of the inverse problem of differential Galois theory: given $G$ and $R$, can one find a structure of $\mathcal{D}_{\mathcal{K}}$-module $M$ on $V$ and a $K$-isomorphism $i: G^{K}(M) \rightarrow G$ such that $R \circ i$ is the natural inclusion of $G^{K}(M)$ in $G L(V)$ ? If it has a positive answer, we say that $G$ is realizable (see [M-S], 2.3, in the standard case), and that the corresponding solutions $M$ realize $(G, R)$, or less precisely, $G$. But this formulation of the problem is too naive. It immediately follows from the discussion of $\S 1$ that

a realizable $K$-group admits a constant group among its inner $K$-forms.

The first constraints listed below for a $K$-group $G$ to be realizable are mere variations on this theme.

1st constraint: a realizable $K$-group $G$ must be isoconstant, i.e. isomorphic over $\bar{K}$ to a constant group. Indeed, an intrinsic differential Galois group $G^{K}(M)$ is a $K$-form of the constant group $G_{K}^{\omega}(M)$.

Of course, this condition is automatically fulfilled if $G$ is a reductive group (cf. [B]), but it is a non trivial one if $G$ is unipotent. For instance, on using the correspondence between unipotent algebraic groups and nilpotent Lie algebras in characteristic 0 , the family of nilpotent Lie algebras given in [Bbk], Ex. 18, §4, yields a counterexample.

2nd constraint: if $G$ is commutative, it must be isomorphic over $K$ to a constant group. Equivalently, its maximal $K$-torus must be split over $K$.

This is clear: a commutative group has only one inner $K$-form.

3rd constraint: we have just seen that a realizable $K$-group $G$ which is non split over $K$ cannot be connected if its connected component of the identity is commutative. In fact, the same conclusion holds true without the latter assumption as soon as the base differential field $K$ is of cohomological dimension $c d(K) \leq 1$ (cf. [S], II.3), as is the field of meromorphic functions on a compact Riemann surface $B$. More precisely:

Proposition 1. Let $G$ be a realizable $K$-group. Assume that $c d(K) \leq 1$, or alternatively that $G^{0}$ is commutative. Then, the degree over $K$ of one of the minimal splitting fields for $G$ divides the number $\left[G: G^{0}\right]$ of connected components of $G \otimes_{K} \bar{K}$.

Proof. Let $M \in \mathbf{D}_{K}$ be a differential equation such that $G=G^{K}(M)$, and let $L=$ $L_{M, \omega}$ be the corresponding Picard-Vessiot extension of $K$. We shall show that in both cases under review, the $K$-group $G^{K}=G^{K}(M)$ is split over the algebraic closure $K^{\prime}$ of $K$ in $L$. Therefore, for one ('the', in the second case) minimal splitting field $F$ for $G$, $[F: K]$ divides $\left[K^{\prime}: K\right]=\left[G: G^{0}\right]$.

Since the condition on $c d(K)$ is stable under finite extensions, and since $G^{K^{\prime}}\left(M \otimes_{K} K^{\prime}\right)$ $\simeq\left(G^{K}(M)\right)^{0} \otimes_{K} K^{\prime}$, we may assume without loss of generality that $K^{\prime}=K$, i.e. that $G^{K}$ is connected. In the second case, we are already done. In the first case, we apply 
Steinberg's theorem (cf. [S], III.2.3, [M-S], 2.2): all torsors under a connected group over such a field $K$ are trivial. Choosing a $K$-rational point on the bitorsor $\mathcal{P}^{K, \omega}$ now yields a $K$-isomorphism between $G^{K}$ and $G_{K}^{\omega}$. Thus $G^{K}$ is constant, hence split over $K$.

REMARK 1. Here is a more conceptual proof of Proposition 1. Since $\Gamma_{K}$ acts trivially on $X^{\omega}=G^{\omega} /\left(G^{\omega}\right)^{0}$, the elements of $H^{1}\left(K, X^{\omega}\right)$ identify with certain equivalence classes in $\operatorname{Hom}\left(\Gamma_{K}, X^{\omega}\right)$, and we deduce from the exact sequence of pointed sets

$$
\{1\}=H^{1}\left(K,\left(G^{\omega}\right)^{0}\right) \rightarrow H^{1}\left(K, G^{\omega}\right) \rightarrow H^{1}\left(K, X^{\omega}\right)
$$

that the class of the $G_{K}^{\omega}$-torsor $\mathcal{P}^{\mathcal{K}, \omega}$, hence of the associated inner form of $G_{K}^{\omega}$, dies over a Galois extension of $K$ whose degree divides the order of $X^{\omega}$. In fact, the exact sequence above gives a bijection $\beta$ between the pointed sets $H^{1}\left(K, G^{\omega}\right)$ and $H^{1}\left(K, X^{\omega}\right)$, cf. [S], III.2.4, cor. 3, so that a more methodological way to formulate the inverse problem is as follows (we assume $c d(K) \leq 1$ ). Let $\mathcal{G}$ be a $C$-group, and let $\mathcal{X}$ be its group of connected components. For any $\xi \in H^{1}(K, \mathcal{X})$, let $\mathcal{G}_{\xi}=\operatorname{Int}\left(\xi^{\prime}\right)$ be the inner form of $\mathcal{G} \otimes_{C} K$ defined by the inverse image $\xi^{\prime}=\beta^{-1}(\xi)$ of $\xi$ in $H^{1}(K, \mathcal{G})$. Under which conditions on $\xi$ is $\mathcal{G}_{\xi}$ realizable? In this point of view, the description given in $\S 1$ of the $K$-structure of $X^{K}$ can be viewed as a constraint on $\operatorname{Int}(\xi)$. On denoting centers by $Z$, and on applying [S], $\S 5$, Prop. 42 , the only freedom left to $\xi$ then lies in $H^{1}(K, Z(\mathcal{X}))$, or more precisely, in the quotient of this group by the image of $H^{1}(K, Z(\mathcal{G}))$.

4th constraint: this does not concern the realizable group $G$ itself, but the differential equations which realize it (in some faithful representation). We assume that $K$ is the field of meromorphic functions on a compact Riemann surface $B$ and denote by $\hat{K}_{s}$ the formal completion of $K$ at a point $s \in B$.

Proposition 2. Let $G$ be a realizable $K$-group, and let $s$ be a point in $B$ such that $G \otimes_{K} \hat{K}_{s}$ is not split over $\hat{K}_{s}$. Then, any $M \in \mathbf{D}_{K}$ realizing $G$ must have a singularity at $s$.

Proof (cf. [K1], 4.1). Otherwise, the torsor $\mathcal{P}^{K, \omega}(M)$ has a $\hat{K}_{s}$-rational point (Cauchy's theorem), and $G^{K}$ becomes isomorphic to the constant group $G_{K}^{\omega}$ over $\hat{K}_{s}$.

REMARK 2. When stating an inverse problem, one usually fixes a set of points $S$ on the base $B$, and insists that the realization $M$ be smooth away from $S$. Proposition 2 shows that the minimal number of singularities needed to solve the intrinsic inverse problem may be larger than the corresponding one (cf. [M-S]) in the standard case. In fact, $S$ will now depend on the $K$-structure of $G$, and it may be useful to view the differential Galois groups as group schemes over $B \backslash S$, as in [A2].

5th constraint: the last condition I want to list concerns $G^{0}$. Although it definitely plays a role (cf. $\S 3$, Remark 3), I apologize for stating it vaguely. Suppose that for some finite extension $K^{\prime}$ of $K, G^{0} \otimes_{K} K^{\prime}$ has been realized by an object $M^{\prime}$ in $\mathbf{D}_{K^{\prime}}$, and that we know that $G$ can only be realized through the differential equation $M$ over $K$ deduced from $M^{\prime}$ by restriction of scalars from $K^{\prime}$ to $K$, i.e. (cf. [K2], 2.7, [A1], II.1.3) that the $C$-representation of $G^{\omega}$ given by $M$ is necessarily induced from the representation given by $M^{\prime}$ of its normal subgroup $\left(G^{\omega}\right)^{0}$. The following result then provides a condition on $G^{0}$. 
Proposition 3. Let $K^{\prime}$ be a finite extension of $K$, let $M^{\prime} \in \mathbf{D}_{K^{\prime}}$, and let $M=$ Res $_{K^{\prime} / K} M^{\prime}$. Then, $G^{K}(M)^{0}$ is contained in the $K$-group $\operatorname{Res}_{K^{\prime} / K}\left(G^{K^{\prime}}\left(M^{\prime}\right)\right)$.

Proof. Since $M^{\prime}$ is a subobject of $M \otimes_{K} K^{\prime}$ in $\mathbf{D}_{K^{\prime}}, G^{K^{\prime}}\left(M^{\prime}\right)$ is a quotient of $G^{K^{\prime}}(M \otimes$ $\left.K^{\prime}\right)$, and we get a $K^{\prime}$-morphism from $\left(G^{K}(M)\right)^{0} \otimes_{K} K^{\prime}$ to $G^{K^{\prime}}\left(M^{\prime}\right)$, hence (cf. [Sp], 12.4.2) a $K$-morphism $\psi:\left(G^{K}(M)\right)^{0} \rightarrow \operatorname{Res}_{K^{\prime} / K} G^{K^{\prime}}\left(M^{\prime}\right)$. Looking at the corresponding maps on the usual Galois groups attached to $\omega, \omega^{\prime}$ as in $\S 1$, we deduce that $\psi$ is injective. See also [A1] II.1.3, where the isomorphism in the last formula should be replaced by an inclusion.

Going back to Remark 1, notice that since $A u t_{\bar{K}}(\mathcal{G})$ acts on the connected component of the identity $\mathcal{G}^{0}$, the cocycle $\xi^{\prime}$ also provides a $K$-form $\mathcal{G}_{\xi}^{0}$ of $\mathcal{G}^{0}$. This gives another interpretation of the group $\left(\mathcal{G}_{\xi}\right)^{0}$ with which Proposition 3 is concerned. When $\mathcal{G}^{0}$ is abelian, this form can be directly described through the natural action of $\mathcal{X}$ on $\mathcal{G}^{0}$, cf. $\S 3$.

We now turn to examples, and check that the simplest possible groups which satisfy the above conditions can indeed be realized as intrinsic Galois groups.

3. Examples in dimension 1. We limit our description to the $C_{1}$-field $K=\mathbf{C}(z)$, with $\partial=d / d z={ }^{\prime}$, to $K$-groups $G$ of dimension 1 and to faithful representations $R$ into $G L_{2}(K)$. We leave the case $G^{0}=\mathbf{G}_{a}$ to the reader. Then, $G^{0}$ is a one-dimensional torus $T$, i.e. the split torus $\mathbf{G}_{m}$ or the circle group $S O(q)$ attached to a non-degenerate binary quadratic form $q$ over $K$. Recall that these anisotropic forms of $\mathbf{G}_{m}$ are parametrized by $H^{1}\left(K\right.$, Aut $\left._{\bar{K}}\left(\mathbf{G}_{m}\right)\right)=H^{1}(K,\{ \pm 1\})=\operatorname{Hom}\left(\Gamma_{K},\{ \pm 1\}=K^{*} /\left(K^{*}\right)^{2}\right.$ : to a non square $\phi \in K^{*}$, one associates the quadratic form $q(x, y)=x^{2}-\phi y^{2}$. The minimal splitting field for $T=S O(q)$ is the quadratic field $K(\sqrt{\phi})$.

We further make the simplest possible assumption taking care of the 3rd constraint simultaneously for all $T$ 's, namely that $X=G / G^{0}$ has order 2 . The only $\Gamma_{K}$-structure $X$ can be endowed with is then the trivial one. In other words, the $K$-groups $G$ we consider are extensions of the constant group $X:=\mathbf{Z} / 2 \mathbf{Z}$ by the $K$-torus $T$. We start by listing all possible such groups.

Let $\tau$ be the homomorphism from $G / G^{0}=\mathbf{Z} / 2 \mathbf{Z}$ to $A u t\left(G^{0}\right)=\{ \pm 1\}$ induced by conjugation. If $\operatorname{Im}(\tau)=\{1\}, G$ is commutative, and by the 2 nd constraint, $T$ must be split. In other words, $G$ must be then the constant group $\mathbf{G}_{m} \times \mathbf{Z} / 2 \mathbf{Z}$. We know that any differential system over $K$ with standard Galois group $\mathbf{C}^{*} \times \mathbf{Z} / 2 \mathbf{Z}$ will realize $G$, say $\left(\begin{array}{l}y_{1}^{\prime} \\ y_{2}^{\prime}\end{array}\right)=\left(\begin{array}{cc}\frac{1}{2 z} & 0 \\ 0 & 1\end{array}\right)\left(\begin{array}{l}y_{1} \\ y_{2}\end{array}\right)$, and $(G, R)$ can easily be realized too. Thus, we assume from now on that $\tau$ is an isomorphism, i.e. that $G$ is not commutative. Notice that when $T$ is isotropic, this forces the restriction of $R$ to $T$ to be isomorphic to the standard diagonal representation of $\mathbf{G}_{m}$ into $S L_{2}$. Since $H^{2}\left(\{ \pm 1\}, \mathbf{G}_{m}\right)$ has two elements, two kinds of groups may then occur:

- If the extension splits, $G$ is the semi-direct product $T \times_{\tau} \mathbf{Z} / 2 \mathbf{Z}$. This is the dihedral group $O T$ attached to $T$ (i.e. when $T=S O(q)$, the orthogonal group $O(q)$ ), and $R$ is isomorphic to its standard representation.

- Otherwise, $G$ contains only one element of order 2 , and is the unique extension $N T$ of $\mathbf{Z} / 2 \mathbf{Z}$ by $T$ which is not a split extension, while $R(G)$ is the normalizer in $S L_{2}$ of its 
Cartan subgroup $R(T)$. (If we replace $T$ by a cyclic group of order 4 , this would give the quaternionic group.)

Here, then, are the images under $R$ of the groups $G$ we are concerned with. The first line indicates the connected component $G^{0}=T$ of $G$; the first column gives the possible split forms $\mathcal{G} \otimes_{C} K$ for $G$, the second one their inner forms. The reason why these inner forms are precisely parametrized by the (outer) forms of $\mathcal{G}^{0} \otimes_{C} K$ on the first line, i.e. by $\phi \in K^{*} /\left(K^{*}\right)^{2}$, was hinted at after Proposition 3: the hypothesis made on $\tau$ gives an identification of $\mathbf{Z} / 2 \mathbf{Z}=\mathcal{G} / \mathcal{G}^{0}$ with $\{ \pm 1\}=A u t\left(\mathcal{G}^{0}\right)$, which is compatible with the natural maps $H^{1}(K, \mathcal{G}) \rightarrow H^{1}\left(K, A u t_{\bar{K}}\left(\mathcal{G}^{0}\right)\right.$ ) (given by inner automorphisms of $\mathcal{G}$ ) and $\beta: H^{1}(K, \mathcal{G}) \rightarrow H^{1}\left(K, \mathcal{G} / \mathcal{G}^{0}\right)($ as in Remark 1$)$.

$$
\begin{aligned}
& \mathbf{G}_{m}=\left\{\left(\begin{array}{cc}
x & 0 \\
0 & y
\end{array}\right), x y=1\right\} ; \quad S O(q)=\left\{\left(\begin{array}{cc}
x & \phi y \\
y & x
\end{array}\right), x^{2}-\phi y^{2}=1\right\} \\
& N \mathbf{G}_{m}=\left\{\left(\begin{array}{cc}
x & 0 \\
0 & y
\end{array}\right),\left(\begin{array}{cc}
0 & x \\
-y & 0
\end{array}\right), x y=1\right\} ; N S O(q)=\left\{\left(\begin{array}{cc}
x & \phi y \\
y & x
\end{array}\right), i\left(\begin{array}{cc}
x & \phi y \\
-y & -x
\end{array}\right), x^{2}-\phi y^{2}=1\right\} \\
& O \mathbf{G}_{m}=\left\{\left(\begin{array}{ll}
x & 0 \\
0 & y
\end{array}\right),\left(\begin{array}{ll}
0 & x \\
y & 0
\end{array}\right), x y=1\right\} ; \quad O(q)=\left\{\left(\begin{array}{cc}
x & \phi y \\
y & x
\end{array}\right),\left(\begin{array}{cc}
x & \phi y \\
-y & -x
\end{array}\right), x^{2}-\phi y^{2}=1\right\} .
\end{aligned}
$$

Realizing $O(q)$ : let $\phi$, with $\operatorname{ord}_{\infty} \phi \in\{-1,0\}$, be a non square element of $K^{*}$, so that $d:=\frac{1}{2} \frac{\phi^{\prime}}{\phi}$ is not a logarithmic derivative in $K^{*}$, and let $M$ be the $\mathcal{D}_{K}$-module structure on $V=K e_{1} \oplus K e_{2}$ given by:

$$
\partial e_{1}=\frac{1}{\phi} e_{2} ; \quad \partial e_{2}=-e_{1}+\frac{1}{2} \frac{\phi^{\prime}}{\phi} e_{2} .
$$

In other words, we are considering the differential equation $y^{\prime \prime}+\frac{1}{2} \frac{\phi^{\prime}}{\phi} y^{\prime}+\frac{1}{\phi} y=0$. Let us show that its intrinsic Galois group $G^{K}=G^{K}(M)$ is $O(q)$, for some quadratic form $q$ associated to $\phi$. We recall that $G^{K}(M)$ is the stabilizer of all the objects of the full tensor category generated by $M$ in $\mathbf{D}_{K}$ :

(i) Since the wronskian equation $w^{\prime}+d w=0$ is not trivial, but its symmetric square is, $G^{K}$ is not connected and its intersection with $S L_{2}$ has index 2 in $G^{K}$.

(ii) The $K$-line generated by the tensor $Q=\phi e_{1}^{2}+e_{2}^{2}$ in $\operatorname{Sym}^{2}(V)$ is stable under $\partial$. Indeed,

$$
\partial Q=\phi^{\prime} e_{1}^{2}+2 \phi e_{1} \partial e_{1}+2 e_{2} \partial e_{2}=\phi^{\prime} e_{1}^{2}+\frac{\phi^{\prime}}{\phi} e_{2}^{2}=\frac{\phi^{\prime}}{\phi} Q .
$$

(In fact, $\frac{1}{\phi} Q$ is an horizontal tensor.) Therefore, $G^{K}$ is contained in $\mathbf{G}_{m} O(q)$, where $q=\phi u^{2}+v^{2}$ denotes a quadratic form in the class of $x^{2}-\phi y^{2}$. In view of (i), $G^{K}$ must then be contained in $O(q)$, but not in $S O(q)$.

(iii) $G^{K}$ is not finite, say because $M$ has an irregular singularity at $\infty$. Hence, $G^{K}$ has dimension at least 1 , and in view of (ii), must coincide with $O(q)$.

The main point in this proof is that in (ii), the tensor $Q$ is not pure, yielding a non-split form of the dihedral group $O \mathbf{G}_{m}$. Of course, the standard differential Galois group $G^{\omega}$ then has no choice but to coincide with the group $O \mathbf{G}_{m}$ over $\mathbf{C}$. For instance, if $\phi=z$, a basis of solutions of the differential equation is given by $\left\{e^{2 i \sqrt{z}}, e^{-2 i \sqrt{z}}\right\}$, and it is easy to check directly that $\operatorname{Aut}_{\partial}\left(K<e^{2 i \sqrt{z}}>/ K\right)=\mathbf{C}^{*} \times_{\tau} \mathbf{Z} / 2 \mathbf{Z}$. 
Realizing $N S O(q)$ : this group can be realized by the tensor product of the previous $\mathcal{D}_{K}$-module $M$ with the rank one equation $\left(\Lambda^{2} M\right)^{-1 / 2}$, i.e. restricting for simplicity to the case $\phi=z$, by the differential equation with basis of solutions $z^{-1 / 4} e^{ \pm 2 i \sqrt{z}}$. Let us give another realization, involving only regular singularities. Let $M$ be the $\mathcal{D}_{K}$-module structure on $V=K e_{1} \oplus K e_{2}$ given by:

$$
\partial e_{1}=\frac{1}{4 z} e_{1}-\frac{1}{z-1} e_{2} ; \quad \partial e_{2}=\frac{1}{z(z-1)} e_{1}-\frac{1}{4 z} e_{2} .
$$

In other words, we are considering the differential system

$$
\left(\begin{array}{l}
y_{1}^{\prime} \\
y_{2}^{\prime}
\end{array}\right)=\left(\begin{array}{cc}
-\frac{1}{4 z} & -\frac{1}{z(z-1)} \\
\frac{1}{z-1} & \frac{1}{4 z}
\end{array}\right)\left(\begin{array}{l}
y_{1} \\
y_{2}
\end{array}\right) \text {. }
$$

Let us show that its intrinsic Galois group $G^{K}=G^{K}(M)$ is $N S O(q)$, with $q$ in the class of $x^{2}-z y^{2}$ :

(i) The wronskian is now trivial, so that $G^{K}$ lies in $S L_{2}$.

(ii) The $K$-line generated by the tensor $Q=e_{1}^{2}+z e_{2}^{2}$ in $\operatorname{Sym}^{2}(V)$ is stable under $\partial$. Indeed,

$$
\partial Q=2\left(\frac{1}{4 z} e_{1}^{2}-\frac{1}{z-1} e_{1} e_{2}\right)+e_{2}^{2}+2 z\left(\frac{1}{z(z-1)} e_{1} e_{2}-\frac{1}{4 z} e_{2}^{2}\right)=\frac{1}{2 z}\left(e_{1}^{2}+z e_{2}^{2}\right) .
$$

Therefore, $G^{K}$ is contained in $\mathbf{G}_{m} O(q)$, and in view of (i), in $\mathbf{G}_{m} O(q) \cap S L_{2}=N S O(q)$. (This reflects the existence a horizontal tensor on this line, namely $\frac{1}{\sqrt{z}} Q$, once the scalars have been extended to the splitting field for $S O(q)$.)

(iii) Here, $M$ is fuchsian. But the local monodromy around 1 has infinite order, so $G^{K}$ has dimension at least 1 , while the local monodromies around 0 and $\infty$ have finite order. Therefore, $G^{K}$ is not commutative, hence cannot lie in $S O(q)$, and in view of (ii), must coincide with $N S O(q)$. (In particular, the standard Galois group $G^{\omega}$ of $M$ is $N \mathbf{G}_{m}$.)

Not realizing $O \mathbf{G}_{m}$, nor $N \mathbf{G}_{m}$, through $R$ : as already pointed out, the restriction of the representation $R$ to the connected component $G^{0}$ of such a group $G$ is the direct sum of a character of $\mathbf{G}_{m}$ and of its inverse, while $R(G)$ permutes the corresponding lines. So, if $M$ realizes $(G, R)$, there exists a quadratic extension $K^{\prime} / K$ with Galois group $G^{\omega}(M) /\left(G^{\omega}(M)\right)^{0}$ such that $M \otimes_{K} K^{\prime}$ is a direct sum of two dual (and non trivial) objects $M^{\prime}, M^{\prime \prime}$ of rank 1 in $\mathbf{D}_{K^{\prime}}$. These spaces cannot be defined over $K$, since $G$ stabilizes no $K$-line. They are therefore conjugate under $\operatorname{Gal}\left(K^{\prime} / K\right)$, and their product in $S y m^{2} M$ is an object $S$ of $\mathbf{D}_{K}$, which contains no non-zero pure $K$-tensor. Not being isotropic, the stabilizer of $S$ cannot contain $R(G)$, which is therefore not realizable.

REMARK 3. Here is a tentative argument to discard these split groups from the list of 'reasonable' $K$-groups in the intrinsic inverse problem: since $M^{\prime}$ and $M^{\prime \prime}$ are not isomorphic over $K^{\prime}$, we deduce from [K2], 2.7.4, that $M$ is induced from $M^{\prime}$, i.e. isomorphic to $\operatorname{Res}_{K^{\prime} / K} M^{\prime}$. By Proposition 3 of $\S 2, G^{0}$ should then be contained in $\operatorname{Res}_{K^{\prime} / K} \mathbf{G}_{m / K^{\prime}}$, but not in the $K$-split subgroup $\mathbf{G}_{m / K}$ given by the homotheties (again because $M \otimes_{K} K^{\prime}$ is not isotypical). By [Sp], 12.4.7.(2), $G^{0}$ must then be isomorphic to the non split $K$-form of $\mathbf{G}_{m / K}$ corresponding to the quadratic extension $K^{\prime} / K$, and $G$ cannot be realized. In 
spite of its incomplete formulation, one can thus consider that $O \mathbf{G}_{m}$ and $N \mathbf{G}_{m}$ violate the last constraint of $\S 2$, and do not provide a negative answer to the intrinsic inverse problem.

In conclusion, we point out that although we have yet to meet a group admitting both split and non split realizations ${ }^{(2)}$, the above examples do show that $G^{K}(M)$ is a sharper invariant than $G^{\omega}(M)$ in the classification of differential equations: let just $\phi$ run through $K^{*} /\left(K^{*}\right)^{2}$. Of course, such possibilities do not occur over base fields of the type $K=\mathbf{C}((z))$, so that in general, higher dimensional examples will be needed to shed light on the link between the finite group $\operatorname{Gal}\left(K^{\prime} / K\right)$, and the $K$-structures of $G^{K}$ and $\left(G^{K}\right)^{0}$.

Acknowledgements. I thank Z. Hajto and T. Crespo for allowing a discrepancy between the titles of my talk ${ }^{(3)}$ and paper (and for the very nice atmosphere of the Będlewo conference), F. Ulmer for having offered me the opportunity to present the above examples at a Rennes, 1996, conference, M. Singer and A. Pillay for their insistence on my writing them down, and Y. André and J. Oesterlé for several enclearing discussions on these topics.

Added in proof. In the style of $\S 2$, Remark 1 , here is a cohomological explanation for the non-realizability of the split groups $G=O \mathbf{G}_{m}, G=N \mathbf{G}_{m}$ of $\S 3$ as intrinsic Galois groups (and more precisely, for the non occurence of $\mathbf{G}_{m / K}$ as their connected component of the identity). Recall the bijection

$$
\beta: H^{1}(K, G) \rightarrow H^{1}\left(K, G / G^{0}\right) .
$$

Since the group $G^{0}=\mathbf{G}_{m}$ is its own centralizer in both $G^{\prime}$ 's, a cocycle $\xi^{\prime} \in H^{1}(K, G)$, mapping in $H^{1}\left(K, A u t\left(G^{0}\right)\right)$ to the class of its trivial outer form, must come from the trivial class $\beta\left(\xi^{\prime}\right)=\xi \in H^{1}\left(K, G / G^{0}\right)=H^{1}\left(K, \mathcal{S}_{2}\right)$; but the rank $2 K$-algebra which such a $\xi$ parametrizes is not a field, and can therefore not be realized as an intermediate Picard-Vessiot extension $K^{\prime} / K$.

More generally, let $K$ be a differential field with $c d(K) \leq 1$, let $\mathcal{X}$ be a finite quotient of its absolute Galois group $\Gamma_{K}$, and let $\mathcal{G}$ be a $C$-group with group of connected components $\mathcal{G} / \mathcal{G}^{0} \simeq \mathcal{X}$. Then, the constant form $G=\mathcal{G} \otimes_{C} K$ of $\mathcal{G}$ is realizable if and only if the center $Z(\mathcal{G})$ of $\mathcal{G}$ maps onto the (necessarily abelian) group $\mathcal{X}$-in which case no other $K$-form of $G$ will be realizable - while the constant form $\mathcal{G}^{0} \otimes K$ of $\mathcal{G}^{0}$ occurs in a realizable $K$-form of $G$ if and only if the centralizer of $\mathcal{G}^{0}$ in $\mathcal{G}$ maps onto $\mathcal{X}$.

\section{References}

[A1] Y. André, Quatre descriptions des groupes de Galois différentiels, in: Springer LN 1296, 1987, 28-41.

${ }^{(2)}$ See however the final comment below on the occurrence of constant forms. I thank J. Hartmann for her remarks on these points.

${ }^{(3)}$ Blended extensions and differential Galois groups: see Unipotent radicals of differential Galois groups, Math. Ann. 321 (2001), 645-666; and Extensions panachées et dualité, Prépubl. Institut de Mathématiques de Jussieu, 287, 2001. 
[A2] Y. André, Différentielles non commutatives et théorie de Galois différentielle ou aux différences finies, Ann. Scient. Éc. Norm. Sup. 34 (2001), 685-739.

[B] D. Bertrand, Review of "Lectures on Differential Galois Theory" by A. Magid, Bull. AMS 33 (1996), 289-294.

[Bbk] N. Bourbaki, Groupes et algèbres de Lie, Chap. I, Masson, 1982.

[Br] L. Breen, Tannakian categories, PSPM AMS 55 (1994), 337-376.

[K1] N. Katz, A conjecture in the arithmetic theory of differential equations, Bull. SMF 110 (1982), 197-239 and 387-88.

[K2] N. Katz, Exponential sums and differential equations, Ann. of Maths Studies 124, Princeton Univ. Press, 1990.

[M-S] C. Mitschi and M. Singer, Connected linear groups as differential Galois groups, J. Algebra 184 (1996), 333-361.

[P] A. Pillay, Finite-dimensional differential algebraic groups and the Picard-Vessiot theory, these Proceedings.

[S] J.-P. Serre, Cohomologie galoisienne, 5e éd., Springer LN 5, 1994.

[Sp] T. A. Springer, Linear Algebraic Groups, 2nd ed., Birkhäuser PM 9, 1998. 